PART VII

\title{
GENERAL DISCUSSION
}




\section{GENERAL DISCUSSION}

Heap: What is the range in excitation among Be shells; and within a given shell, what is the range in excitation?

Cowley and others: The excitation in Be shells varies enormously from star to star. The typical lines seen are due to Fe II, Ti II, etc. and also the hydrogen lines, but in some of the hotter stars you see helium lines ( $\lambda$ 3889, etc.), which are also due to the shell. One can place limits: at the hot end, one does not see $\mathrm{He}$ il shell lines and at the cool end, neutral metallic lines are not usually seen. Within a given shell, the range of excitation is much less.

Conti: I am concerned about the classification of Be stars, because in some cases things are somewhat confusing. There are really several kinds of stars which we are collectively referring to as Be stars. I think we are in a position now where by looking at the spectra we can distinguish between certain types of B-type stars which show emission. I would like to propose that we call a star a Be star if it is a more-or-less main sequence B-type star with emission in the hydrogen lines and sometimes in the helium lines (I do not believe we are at a stage yet where we can distinguish between a single and a binary star so I don't think we can put that into a classification system). A second class of objects would be those B-type stars which show forbidden emission lines and $I$ would suggest that we classify these as $B$ with a small $e$ in brackets $B[e]$, following the notation for forbidden lines. The so-called Herbig Ae and Be stars might all collectively be called Ae stars because most are A types anyway. A distinguishing nomenclature would help us recognize their rather different evolutionary history. The supergiant stars should not be collectively referred to as Be stars at all, as they have emission because of stellar winds. I would suggest that they be called only by their spectral type and luminosity class, which can be followed by a small e, as for example B5Ie, and referred to as supergiants and not as Be stars. The tiny group of $O$ type stars which show central hydrogen emission can be called Oe stars, as I have suggested earlier. I would also propose the small letter $p$ be used only for those objects which do not otherwise fit into these classes. It would be desirable to have some notation for stars which exhibit shells, but we have not yet been able to define a shell star and so I think that we should not include this into our classification scheme at this time.

Garrison: We must consider what to put into classification schemes. It seems to me that it is premature to attempt a detailed classification of Be stars. In lieu of that, I would like to suggest the following: that something like the scheme of Conti be included in the spectral type column of a table and that a fairly complete description of peculiarities be put in the notes to the table, with the following guidelines: Table

(1) Spectral type, luminosity class, rotation ( $\mathrm{n}$ or $\mathrm{nn}$ ), and emission (e). Notes

(2) difference in line width between hydrogen and helium, if any. 
(3) whether hydrogen emission is narrow or broad.

(4) absorption reversal in cores ('shell' formerly).

(5) Fe II emission or 'shell' absorption.

(6) veiling or apparent filling in of lines.

(7) infrared: $\mathrm{O}_{\mathrm{I}}, \mathrm{Ca}$ II.

(8) ultraviolet: $\mathrm{Si}$ IV, $\mathrm{Mg}$ II, Fe III.

(9) variation and time scale (if known).

(10) line asymmetries (P Cyg, inverse P Cyg, $V / R$, etc.)

Since Be stars are probably all variable, the presence of any of these characteristics at any epoch shows that the particular star is capable of showing them (e.g. only some stars become 'shell' stars).

I would like to emphasize that the detailed description should be relegated to the notes and that, at the present time, we not try to incorporate all of this information into the classification scheme. It may be that some day when we understand Be stars, we will be able to couple several of these characteristics, but attempts to do that now, as with Lesh's system, are bound to fall into disuse (because Fe II is not always coupled with strong hydrogen emission, for example). Also we must avoid a scheme with too many parameters because only the proponent of the scheme will ever be able to remember it (e.g., a well-known galaxy classification scheme).

I am willing to start this with the extensive table of southern OB star classifications that I will be preparing in a few months; i.e., indication of emission in the table with extensive description in the notes at the end of the table.

Snow: My suggestion of a useful note to include among all the other spectroscopic descriptions would be one saying whether or not line asymmetries indicative of mass loss are seen in the ultraviolet, and if so, which lines show mass loss effects.

Schild: I should like to support the suggestion of Dr Garrison. It would be opening Pandora's box to try to expand the present classifications as listed in the spectral type column of a table. At the same time, it is extremely useful to have remarks to such a table, organized in a systematic way, so that a person desiring to make further observations of a class of stars (such as all stars showing Fe II emission, or sharp He I absorption, or possibly veiling of the $\mathrm{He}_{\mathrm{I}}$ spectrum) could easily select a list of objects. It is particularly useful if the information is organized in a systematic way.

Cowley: What observational quantities do the theorists need?

Hummer: It is important to try to make as much use of continuum information as possible. Well selected, high accuracy profiles are also important. I think that low dispersion material is almost useless for model making. It is important to get a wide variety of lines and to get some photospheric lines as well as shell lines, so that we can get some idea of what the inside of the star looks like as well as the outside.

Marlborough: I agree with everything that Hummer says. It is important to give data which are quantitative instead of just qualitative. I also support Hummer's suggestion that continuum data are very useful. Also, it is important to have accurate line profiles as Hummer suggested - that is, lines of different excitation potentials, shell lines, emission lines, and stellar lines, all with the highest dispersion possible. Simultaneous photometry would also be useful, especially if the stars vary. Narrow band interference filter photometry would help to determine whether the lines vary, whether the continuum varies, or whether both vary. 
Hummer: Speaking as an ignorant theoretician it would be useful for those of us involved in model building if you could give examples of a well observed, well behaved, non-varying, classical Be star.

Cowley: 1 Del.

Schild: $\chi$ Oph.

Someone: But the polarization varies.

Heard: If you insist on a star which does not vary, you might end up picking the most unusual kind of Be star.

Hummer: How about a well behaved shell star?

Schild: Pleione is typical. It loses the shell, the shell dissipates, it becomes a Be star and then it becomes a normal garden-variety, main-sequence B8 star. It is in a cluster. Even though it is variable, Pleione is a universal Be star.

I seem to recall that many of McLaughlin's observations of $V / R$ variations do not show strict periodicity, but rather show quasi-periodicity. Does not the binary star hypothesis for Be stars require exact periodicity, even though the amplitude of the effect may vary? Can the binary star proponents comment?

Harmanec: It is necessary to consider two types of $V / R$ variation. The first type may last days or up to several hundred days and is strictly periodic. But there is also a 5-50 year variation which may be related to the slow rotation of an elliptical ring, formed during a non-continuous process of mass transfer around the mass-gaining component. This type of variation need not be periodic but may be simply cyclic instead, reflecting thus a gradual circularization of the elliptical ring.

Hutchings: If large amounts of mass are being transferred would you not expect to see peculiar abundances because you are coming right down to nuclear processed material? (as e.g., in the supergiant binary HD 163181).

Plavec: I do not think you would recognize too many changes. You may remember that Bolton in Cambridge claims that the anomalous abundances in certain $\mathrm{O}$ and $\mathrm{B}$ stars might be due to this, but $\mathrm{I}$ do not think you can expect too much.

Poeckert: For $\zeta$ Tau or $\phi$ Per, which have periods of 100 days or more, would $V / R$ variations which are less than the orbital period be a problem in the binary hypothesis?

Plavec: Yes. If we are certain that the orbital period in $\phi$ Per is 130 days, say, and a $V / R$ variation of 80 days were observed, it would be a problem for the binary hypothesis.

Peters: What are $V / R$ variations? For a number of years, I have been attempting to determine the cause of this type of profile variation. In the case of $H R 2142, V / R$ variations can be understood in terms of variable absorption components on the blue sides of the emission line profiles.

How many high dispersion observations of $\mathrm{H} \alpha$ and $\mathrm{H} \beta$ exist for Be stars and what is the extent of the time coverage? Many older observations did not show structure because the plates were of too low a dispersion. Usually, only the instrumental profile (a Gaussian) was observed.

Hutchings: In Victoria, we have high quality photographic, photoelectric, and TV profiles of some bright Be stars. However, coverage is sporadic and irregular. I imagine the same is true at, say, Haute Provence or Lick or Perkins.

Plavec: We heard it said at this Symposium that some of the stars observed with 
the Copernicus satellite show a different velocity of rotation as derived from the lines in the ultraviolet as compared with rotations derived in the normal visual region. I think that this is a very exciting fact; I would like to hear more about this and if possible some explanation.

Snow: I have noticed one or two cases of this, though these are not Be stars. One example is 42 Ori, a B1V star, which shows lines sharper in the ultraviolet than you would expect from $v$ sin $i$ 's based on visual spectra.

Peters: I wonder if this effect is not confined to the shell stars, because I do not find this effect in the 'classical' Be stars which I observed, $v$ Cyg or $\mu$ Cen.

Heap: Most of the ultraviolet stellar lines that are most useful have broad wings (damping wings) and so one does not want to just measure the full widths of the lines and say, "Ah, I have a rotational velocity". I think one must compare the observed profiles against theoretical profiles to estimate rotational velocity. I have computed a grid of profiles for Si III, Si IV, C III, and C IV lines, for temperatures from 17000 to $30000^{\circ}$. In the case of $\zeta$ Tau, which is the only star I have looked at, I found that the rotational velocity is the same regardless of which line I looked at in the ultraviolet. And this bothers me. I would have thought that $\mathrm{Si}$ III $\lambda$ 1206, being a lower excitation line than $\mathrm{Si}$ IV $\lambda 1393, \lambda 1403$, would be formed nearer to the equator and would therefore have a higher projected rotational velocity, but that was not the case.

Plavec: And the rotational velocities you derived are significantly lower in the ultraviolet than in the ordinary blue region?

Heap: Yes.

Hutchings: I might suggest that if you are looking at a star with a temperature gradient across its surface, in the far ultraviolet the underlying continuum radiation may come from the polar regions, where one would expect $v \sin i$ to be small. You may then expect to see a dependence of $v \sin i$ on the line wavelength.

Poeckert: Perhaps the theoreticians should get to work and calculate some ultraviolet line profiles to compare with the visual ones.

Heap: I have computed profiles for a spherically symmetric model. Gravitydarkened profiles for ultraviolet lines are badly needed.

Cowley: One thing that is very important to do now is to have very careful photometry. It seems to me that what we know is that the Be stars are variable but that is about all we know. Especially for the systems where there is some evidence that they are binary, we need good photometry, perhaps in one or two cycles. It is not good enough to have an observation now and one three months from now and one three years from now and to try to put them together because there may be erratic variations from cycle to cycle.

Feinstein: It would be most desirable to have simultaneous spectroscopy and photoelectric photometry.

Slettebak: I would like to raise two questions which I brought up earlier in this Symposium. The first goes back to the single star hypothesis. Bidelman raised the question explicitly in his summarizing remarks as to how the ring is formed. I would like to ask the theoreticians present whether they have any ideas with regard to the pulsational hypothesis which I mentioned earlier as a trigger to get material into the ring, and whether such a process might be feasible. My second question has to do with the fact that very broad $\mathrm{H} \alpha$ emission is observed in some Be stars, much broader than 
the absorption lines. How does one understand this? Electron scattering and turbulence have been suggested - do you have any ideas about this?

Harmanec: There was a paper by Morgan in the Astrophysical Journal $(195,391$, 1975 ) in which he computed some hydrodynamics in rotating single stars. I would like to hear more about this work.

Marlborough: What he did was to perform a linear stability analysis of Limber's hydrostatic solutions and looked for unstable temporal and angular modes. He found that he could obtain unstable modes with a variety of periods and, in particular, he could get variations with time scales of the order of a few minutes. Now there was no consideration of what could initiate such variations, so he was only showing that instabilities could grow and you could get variations with this time scale. So it is possible that circumstellar envelopes around single stars have certain instabilities in them which might be able to account for some of the variations people have seen, but in all these linear stability analyses you do not know whether the perturbations will grow or not unless you go to the non-linear theory. But at least certain modes are unstable. With regard to the large $\mathrm{H} \alpha$ emission widths, I suggested long ago that these might be due to electron scattering in the inner regions of a very dense disk, but no one has made any calculations along these lines. But the densities are high enough and the electron velocities are large enough at, say $10000^{\circ}$, to qualitatively explain this effect.

Hummer: Auer and Mihalas (Astrophys. J. 153, 245, 923, 1968) have done calculations with electron scattering for O-type stars to see how well electrons do really broaden lines. They found that because the electron scattering cross section is so very small, under many conditions the continuous absorption gets most of the photons before they can be scattered by electrons.

Coyne: Could someone give a list of five stars which would be the most interesting for someone to get a light curve for?

Peters: HR 2142.

Plavec: 4 Her.

Herman: I would like to say that our photographic catalogue of $250 \mathrm{Be}$ stars arranged according to their classification by the intensity and depth of the hydrogen lines, will be published next year. 